dysplasia (IED). The patient received parenteral and enteral nutrition with elemental formulas.

Previous reports have suggested that IED may be a congenital inherited autosomal recessive disease. We report a case of congenital enteropathy that represents a diagnostic and therapeutically challenge.

\section{EOSINOPHILIC DIGESTIVE DISEASE AND ATOPIC BRONCHIAL ASTHMA; TWO DISEASES OR AN EXPRESSION OF ONE DISEASE IN TWO DIFFERENT SYSTEMS}

doi:10.1136/archdischild-2012-302724.0707

M Yakoot. Internal Medicine and Pediatrics, Green Clinic and Research Centre, Alexandria, Egypt

Eosinophilic digestive disease (EDD) includes a broad spectrum of clinical presentations due to eosinophilic inflammation involving anywhere from the esophagus to the rectum. The heterogeneity in the clinical presentations of EDD is determined by the site and depth of eosinophilic infiltration. The sites of inflammation determine the nomenclature for EDD. The most well characterized of these, eosinophilic esophagitis (EE), eosinophilic gastroenteritis (EG), and eosinophilic colitis or enterocolitis. While the depth of esosinophilic infiltration through the three main layers (mucosa, musculosa and serosa) determines the prominent clinical manifestation. The recent advances in gastrointestinal endoscopy and the increasing awareness and diagnosis of EDD, in my viewpoint, can be of help to add to our understanding of the heterogeneous clinical syndrome under the broad title bronchial asthma.

Here I present a multidisciplinary comparitive analysis to prove that EDD and the allergic bronchial asthma can be regarded as two clinical expressions of one disease in two systems that are functionally different but anatomicaly and embryologically related.

\section{PREVALENCE OF FAT-SOLUBLE VITAMIN DEFICIENCIES IN CHILDREN WITH CYSTIC FIBROSIS}

doi:10.1136/archdischild-2012-302724.0708

AK Gupta, V Ng, J Garside. Paediatrics, Calderdale and Huddersfield NHS Trust, Halifax, UK

Background Malabsorption of fat-soluble vitamins is likely in most patients with cystic fibrosis, particularly those who are pancreatic insufficient. Low vitamin levels are associated with poorer clinical status, and reduced lung function. Since the introduction of improved pancreatic enzymes, normal to high fat diets and routine vitamin supplementation, clinical evidence of fat-soluble vitamin deficiency is rarely seen.

Aim To determine the prevalence of fat-soluble vitamin deficiencies in children with cystic fibrosis in Calderdale and Huddersfield NHS Trust.

Method Retrospective analysis of vitamin levels performed in children with cystic fibrosis in Calderdale and Huddersfield Trust over a period of six years. Data was available for 19 out of 22 children with cystic fibrosis. Results of plasma vitamin A, D (Total $25 \mathrm{OH}$ Vit D) and $\mathrm{E}$ levels for these children were collected on a pre-designed proforma.

Results Vitamin A, D and E levels were checked for a total of 75, 75 and 76 times respectively over a period of six years. Vitamin E levels were normal. Vit A levels were subnormal on two occasions. However, Vitamin D levels were sub-optimal (<60nmol/1) on forty occasions and amongst them levels were below $20 \mathrm{nmol} / \mathrm{l}$ on three occasions.

Conclusion Sub-optimal Vitamin D levels are still very common in children with cystic fibrosis despite routine vitamin supplementation.

\section{SPREADING OF NOURISHMENT DISORDERS IN SCHOOLCHILDREN POPULATION}

doi:10.1136/archdischild-2012-302724.0709

${ }^{1} \mathrm{~T}$ Zerekidze, ${ }^{2} \mathrm{~N}$ Adamia, ${ }^{3} \mathrm{M}$ Surviladze, ${ }^{1}$ Chkhaidze, ${ }^{2} \mathrm{~N}$ Katamadze, ${ }^{3} \mathrm{M}$ Kartozia, ${ }^{4} \mathrm{D}$ Khachapuridze, ${ }^{3} \mathrm{~V}$ Kherkheulidze, ${ }^{1} \mathrm{M}$ Gugunishvili. ${ }^{\top}$ Tbilisi State Medical University; ${ }^{2}$ Department of General Pediatrics, Tbilisi State Medical University; ${ }^{3} \mathrm{M}$. Iashvili Pediatric Clinic, Tbilisi; ${ }^{4}$ Kutaisi Pedagogical University, Kutaisi, Georgia

Goal Study of nourishment disorders' spreading and evaluation of the impact of risk factors in schoolchildren population.

Materials and Methods Study included 3012 children of 6-15 age: 1654 girls and 1358 boys. By means of the questionnaire we studied prevalence of nourishment disorders among schoolchildren population; evaluated peculiarities of clinical course and mathematically evaluated frequency and combinations of risk-factors. Statistical processing was provided by SPSS/v.12 software.

Research results Study was conducted by stages. In $47.2 \%$ of cases set of gastroezophagical symptoms was identified. Parents of $41 \%$ of the questioned children had gastrointestinal pathologies. Together with routine studies, $\mathrm{pH}$-metry, measurement of amylase and lipase levels in blood and urine was conducted.

Studies showed various organic pathologies in $57 \%$ of children, among them: gastric and duodenal ulcer in $9 \%$ of cases, pancreatitis, reflex-esophagitis, associated with ulcer in 19\% of cases, diaphragm hernia in $8 \%$.

In $48 \%$ of cases organic pathologies, recurrent diseases, congenital disorders were excluded. Mentioned population had the diagnosis of functional dyspepsia, with the further relevant treatment scheme and diet.

Conclusion Data of our epidemiological studies do not significantly differ from the data of various countries and in our case the contributing factor is Georgian cuisine.

\section{MAGNETIC RESONANCE IMAGING VERSUS ULTRASONOGRAPHY IN ASSESSING CHANGES IN FAT LIVER CONTENT IN OBESE CHILDREN AFTER ONE-YEAR NUTRITIONAL INTERVENTION}

doi:10.1136/archdischild-2012-302724.0710

'E Verduci, ${ }^{2} \mathrm{C}$ Pozzato, 'G Radaelli, ${ }^{2} \mathrm{~A}$ Rovere, 'M Salvioni, 'G Banderali, 'E Riva, 'M Giovannini. 'Department of Pediatrics; ${ }^{2}$ Department of Radiology, University of Milan, Milan, Italy

Background and Aims Despite the potential clinical and practical relevance, there is lack of studies in current literature assessing the relationship of longitudinal change of liver fat content with liver biochemical parameters in paediatric age. The aim of the present study was to assess whether any association may exist of change in liver fat content based on MRI with change in liver biochemical parameters in obese children who underwent a one-year nutritional intervention.

Methods Fourthy six obese children, aged 6-14 years, underwent metabolic measurements, liver ultrasonography (US) and chemicalshift MRI examinations at baseline and after an one-year nutritional intervention. Biochemistry included serum alanine aminotransferase (ALT) and aspartate aminotransferase (AST). Liver fat fraction (FF) on MRI was judged elevated as it was $39 \%$.

Results Prevalence of FF3 $9 \%$ declined from $34.8 \%$ to $8.7 \%(P<0.01)$, with a mean $(95 \% \mathrm{CI})$ reduction of $7.8(5.0-10.6) \%$. At baseline, FF was associated with any liver biochemical parameters (maximum $P<0.001)$. At the end of intervention association was found with AST $(P=0.017)$. Change of FF was associated with change in AST $(P=0.027)$ and $\operatorname{ALT}(P=0.024)$. Liver echogenicity was associated with ALT at baseline $(P<0.001)$. An age and sex adjusted multiple regression analysis showed that FF change was independently associated with change in serum AST (adjusted regression coefficient 0.348, $P=0.048$ ). 
Conclusions The results suggest that in obese children undergoing nutritional interventions longitudinal changes in liver fat content may be associated with change in serum transaminases suggesting novelty in monitoring NAFLD in childhood obesity.

\section{COMPARISON BETWEEN OVERWEIGHT/OBESE (OW/OB) PATIENTS: COELIAC VS NON COELIAC ONES}

doi:10.1136/archdischild-2012-302724.0711

R Mercurio, D Guttadoro, M Podagrosi, A Grimaldi, M Barbato, AM Caiazzo, L Petrarca, A Vania. Dept of Paediatrics and Paediatric Neuro-Psychiatry, 'Sapienza' University of Rome, Rome, Italy

Background and Aims In literature there are case reports of coeliac disease (CD) diagnosed in $O W / O B$ children and studies assessing the body weight excess (EBW) at CD diagnosis. Besides, an incorrect gluten-free-diet (GFD) often leads to EBW. Aim of this study is to assess the effect of a balanced diet in coeliac patients (CP) with EBW compared to matched non-coeliac patients (NCP) with EBW.

Methods and results: In 2006-2010, we selected 8 (4M, 4F, age $=11.35 \pm 3.79$ ) out of $29 \mathrm{CP}$ we follow; they all had EBW $(z-B M I=1.93 \pm 0.69)$ and were compliant to the GFD. Follow-up lasted $11.62 \pm 1.68$ months, with a $z$-BMI reduction in all cases (z-BMI=1.45 \pm 1.01$)$. Among the matched 8 NCP (4M, 4F, age $=10.52 \pm 3.60$; start-up's $z-B M I=2.07 \pm 0.59$;), followed-up for the same period of time, 7 reduced their $z-B M I(1.57 \pm 0.67)$, while in 1 it worsened.

Conclusions Despite the limited number of patients, our study underlines the importance of a strict supervision in CP with EBW to improve their nutritional status. We also noticed that the coherent adhesion to a balanced GFD and the consequent effect on z-BMI are better than those among NCP, probably because in CP the nutritional prescriptions are perceived as indispensable for the therapy of CD.

\section{Bibliografia:}

Mariani P et al. J Pediatr Gastroenterol Nutr 1998; 27:519-523;

Polito C et al. Nutr Res 1992; 12:353-358;

Rea F et al. J Pediatr Gastroenterol Nutr 1996; 23:408-412;

Dickey W, Kearney N. Am J Gastroenterol 2006; 101:2356-2359;

Aydogdu S et al. Dig Dis Sci 2009; 54:2183-2187;

Patro B, Szajewska H. Curr Opin Clin Nutr Metab Care. 2010; 13:300-4

\section{SAFETY AND EFFICACY OF OMEGAVEN IN PRETERM NEONATES WITH PARENTERAL NUTRITION ASSOCIATED LIVER DISEASE}

doi:10.1136/archdischild-2012-302724.0712

${ }^{1} \mathrm{G}$ Booth, 'T Havranek, 'E Armbrecht, ${ }^{2} \mathrm{M}$ Revenis, ${ }^{2} \mathrm{C}$ Klein, ${ }^{2} \mathrm{~L}$ Scavo. ${ }^{1}$ Saint Louis University, St. Louis, MO; ${ }^{2}$ George Washington Univ., Children's National Med Center, Washington, DC, USA

Background Omegaven, a fish oil derived parenteral lipid emulsion rich in omega-3 fatty acids, has been used in the United States under Investigational New Drug applications to treat parenteral nutrition associated liver disease (PNALD) in neonates.

Aims To evaluate the safety profile and changes in serum direct bilirubin levels in preterm neonates with moderate to severe PNALD treated with Omegaven

Methods A prospective observational study of parenteral nutrition (PN) dependant neonates with consecutive serum direct bilirubin levels above $3 \mathrm{mg} / \mathrm{dL}$, separated by a minimum of 7 days, who were expected to require PN for at least 3 more weeks were considered for Omegaven therapy. Eligible neonates were treated with Omegaven, as the only lipid infusion, at a maximum rate of $1 \mathrm{~g} / \mathrm{kg} /$ day. Omegaven therapy was stopped once the serum direct bilirubin level was less than $1 \mathrm{mg} / \mathrm{dL}$ on consecutive weeks, or the patient tolerated adequate enteral nutrition to stop PN. The change in serum direct bilirubin was compared using the Wilcoxon signedrank test.

Results 17 neonates were eligible for the analysis with a mean gestational age at birth of 26.6 weeks and mean birth weight of $911 \mathrm{~g}$. Omegaven was administered for a median of 57 days. The neonates tolerated Omegaven well, without significant changes noted in the safety indicators (coagulation profile, platelet count, serum triglyceride, glucose, albumine).

Serum direct bilirubin levels decreased significantly during the treatment course $(p=0.035)$.

Conclusions Significant decrease in serum direct bilirubin levels was observed in neonates with PNALD; omegaven was well tolerated.

\section{COMPARISON BETWEEN PROPOFOL VERSUS PROPOFOL- KETAMINE COMBINATION IN PAEDIATRIC UPPER GASTRO- INTESTINAL ENDOSCOPY}

doi:10.1136/archdischild-2012-302724.0713

${ }^{1} 0$ Genovese, ${ }^{2} \mathrm{~A}$ Chiaretti, ${ }^{1} \mathrm{~A}$ Mancino, ${ }^{2} \mathrm{~A}$ Gatto. ${ }^{1}$ Paediatric Intensive Care Unit; 2Pediatrics, Policlinico Gemelli University Hospital, Roma, Italy

Objective To assess the safety and effectiveness of the ketamine plus propofol combination versus propofol alone for procedural sedation in pediatric patients undergoing upper GI endoscopy.

Patients and design: A retrospective case series of patients undergoing procedural sedation for upper GI endoscopy was studied. Median age was $6.3(0.4-13.1)$ years. 29 patients received propofol alone while 37 patients received propofol plus ketamine; the child's degree of sedation was scored using the modified Ramsay sedation scale. Procedural success (length of the procedure), adverse effects, recovery time, and vital signs were also measured.

Results No patient in either group experienced major adverse events or required tracheal intubation. In group receiving ketamine and propofol, the total dose of propofol required was significantly lower than in Propofol alone (3.4. $\pm 3.2 \mathrm{mg} / \mathrm{kg}$ vs. $5.2 \pm 3.4 \mathrm{mg} / \mathrm{kg}$; $\mathrm{P}<0.001)$. The incidence of hypotension was also significantly lower (10\% vs. $37 \%$; $\mathrm{P}<0.001)$. Both best analgesia and shorter recovery time were obtained with the propofol-ketamine association. No differences were observed in the degree of sedation and in the awakening quality score between the two groups.

Conclusions The combination of propofol and ketamine provides a good solution for procedural sedation in during GI endoscopic procedures. Compared to propofol alone, ketamine and propofol results in less hypotension and reduces the incidence of respiratory depression.

\section{RELATIONSHIP BETWEEN ESOPHAGEAL SWALLOW PATTERNS AND REFLUX CHARACTERISTICS IN NEWBORNS WITH SYMPTOMS OF GASTROESOPHAGEAL REFLUX DISEASE}

doi:10.1136/archdischild-2012-302724.0714

${ }^{1} \mathrm{~F}$ Cresi, ' $\mathrm{SA}$ Liguori, ${ }^{\mathrm{E} E}$ Locatelli, 'E Maggiora, ${ }^{2} \mathrm{~F}$ Savino, 'E Bertino. ${ }^{1}$ Neonatal Intensive Care Unit, Department of Paediatrics; ${ }^{2}$ Dep. of Pediatrics, University of Torino, Torino, Italy

Background and Aims Transient lower esophageal sphincter relaxations are primarily responsible for GER in healthy infants and plays an important role in preterm and term infants with GER disease (GERD). Gastroesophageal dismotility due to immaturity could also promote impaired swallowing patterns influencing the esophageal clearance. The aim of this study was to investigate the relationship between esophageal swallow patterns and reflux characteristics in newborns with GERD symptoms. 